\title{
An approach to exact solutions of the time-dependent supersymmetric two-level three-photon Jaynes-Cummings model
}

\author{
Jian-Qi Shen*, Hong-Yi Zhu and Hong Mao \\ State Key Laboratory of Modern Optical Instrumentation, Center for Optical \\ and Electromagnetic Research, College of Information Science and Engineering \\ Zhejiang Institute of Modern Physics and Department of Physics, \\ Zhejiang University, Hangzhou 310027, People's Republic of China
}

(October 31, 2018)

\begin{abstract}
By utilizing the property of the supersymmetric structure in the two-level multiphoton JaynesCummings model, an invariant is constructed in terms of the supersymmetric generators by working in the sub-Hilbert-space corresponding to a particular eigenvalue of the conserved supersymmetric generators. We obtain the exact solutions of the time-dependent Schrödinger equation which describes the time-dependent supersymmetric two-level three-photon Jaynes-Cummings model (TLTJCM) by using the invariant-related unitary transformation formulation. The case under the adiabatic approximation is also discussed.

Keywords: Supersymmetric Jaynes-Cummings model; exact solutions; invariant theory; geometric phase factor; adiabatic approximation
\end{abstract}

\section{INTRODUCTION}

The interaction between a two-level atom and a quantized single-mode electromagnetic field can be described by the Jaynes-Cummings model (JCM) [1] which has been applied to investigate many quantum effects such as the quantum collapses and revivals of the atomic inversion, photon antibunching, squeezing of the radiation field, inversionless light amplification, electromagnetic induced transparency [2-5], etc.. In addition to the standard JCM, there exists another type of JCM which possesses supersymmetric structure [6,7]. In this generalization of the J-C model, the atomic transitions are mediated by $k$ photons. Singh has shown that this model can be used to study multiple atom scattering of radiation and multiphoton emission, absorption, and laser processes [8]. Some authors introduced a supersymmetric unitary transformation to diagonalize the Hamiltonian of this supersymmetric JCM and obtain the eigenfunctions of the stationary Schrödinger equation $[9,10]$. It is of great interest to investigate the geometric phase factor of the time-dependent JCM since the geometric phase factor [11] appears only in systems with the timedependent Hamiltonian. It can be verified as shown in the present paper that the exact solutions and the geometric phase factor of the two-level JCM whose Hamiltonian has time-dependent parameters can also be obtained by making use of the generalized invariant theory [12]. For simplicity and convenience, in this paper we only investigate the time-dependent supersymmetric two-level three-photon Jaynes-Cummings model (TLTJCM). This method can also be generalized to the time-dependent JCM with more than three photons.

The invariant theory which is appropriate for treating time-dependent systems was first proposed by Lewis and Riesenfeld (L-R) [13] in 1969. In 1991, Gao et al. generalized the L-R invariant theory and proposed the invariantrelated unitary transformation formulation $[12,14]$. In this formulation the eigenstates of the time-dependent invariants are replaced with those of the time-independent invariants through the unitary transformation and the exact solutions (which contain the dynamical and geometric phase factor) of the time-dependent Schrödinger equation are obtained. Many works have shown that the invariant-related unitary transformation approach is a powerful tool for treating time-dependent problems and geometric phase factor [15-17].

\section{THE EXACT SOLUTIONS OF THE TIME-DEPENDENT TLTJCM}

The Hamiltonian of the TLTJCM under the rotating wave approximation is given by

$$
H(t)=\omega(t) a^{\dagger} a+\frac{\omega_{0}(t)}{2} \sigma_{z}+g(t)\left(a^{\dagger}\right)^{3} \sigma_{-}+g^{*}(t) a^{3} \sigma_{+},
$$

*E-mail address: jqshen@coer.zju.edu.cn 
where $a^{\dagger}$ and $a$ are the creation and annihilation operators for the electromagnetic field, and obey the commutation relation $\left[a, a^{\dagger}\right]=1 ; \sigma_{ \pm}$and $\sigma_{z}$ denote the two-level atom operators which satisfy the commutation relation $\left[\sigma_{z}, \sigma_{ \pm}\right]=$ $\pm 2 \sigma_{ \pm} ; g(t)$ and $g^{*}(t)$ are the coupling coefficients and 3 is the photon number in each atom transition process; $\omega_{0}(t)$ and $\omega(t)$ are respectively the transition frequency and the mode frequency. All the parameters in Eq. (2.1) are time-dependent, and the time-dependent Schrödinger equation for this system is

$$
i \frac{\partial|\Psi(t)\rangle_{s}}{\partial t}=H(t)|\Psi(t)\rangle_{s}
$$

The supersymmetric structure can be found in the TLTJCM by defining the following supersymmetric transformation generators $[9,10]$ :

$$
\begin{aligned}
& N=a^{\dagger} a+\sigma_{z}+\frac{1}{2}, \quad N^{\prime}=\left(\begin{array}{cc}
a^{3}\left(a^{\dagger}\right)^{3} & 0 \\
0 & \left(a^{\dagger}\right)^{3} a^{3}
\end{array}\right), \\
& Q=\left(a^{\dagger}\right)^{3} \sigma_{-}=\left(\begin{array}{cc}
0 & 0 \\
\left(a^{\dagger}\right)^{3} & 0
\end{array}\right), \quad Q^{\dagger}=a^{3} \sigma_{+}=\left(\begin{array}{cc}
0 & a^{3} \\
0 & 0
\end{array}\right) .
\end{aligned}
$$

It is easily verified that $\left(N, N^{\prime}, Q, Q^{\dagger}\right)$ form supersymmetric generators and have supersymmetric Lie algebra properties, i.e.,

$$
\begin{aligned}
Q^{2} & =\left(Q^{\dagger}\right)^{2}=0, \quad\left[Q^{\dagger}, Q\right]=N^{\prime} \sigma_{z}, \quad\left[N, N^{\prime}\right]=0, \quad[N, Q]=Q, \\
{\left[N, Q^{\dagger}\right] } & =-Q^{\dagger}, \quad\left\{Q^{\dagger}, Q\right\}=N^{\prime}, \quad\left\{Q, \sigma_{z}\right\}=\left\{Q^{\dagger}, \sigma_{z}\right\}=0, \\
{\left[Q, \sigma_{z}\right] } & =2 Q, \quad\left[Q^{\dagger}, \sigma_{z}\right]=-2 Q^{\dagger}, \quad\left(Q^{\dagger}-Q\right)^{2}=-N^{\prime},
\end{aligned}
$$

where \{\} denotes the anticommuting bracket. By the aid of Eqs. (2.3) and (2.4), the Hamiltonian (2.1) of the TLTJCM can be rewritten as

$$
H(t)=\omega(t) N+\frac{\omega_{0}(t)-2 \omega(t)}{2} \sigma_{z}+g(t) Q+g^{*}(t) Q^{\dagger}-\frac{\omega(t)}{2} .
$$

According to the L-R invariant theory [13], one should first construct an invariant $I(t)$ in order to show the solvability of Eq. (2.2). A Hermitian operator $I(t)$ is called invariant if it satisfies the following invariant equation

$$
\frac{\partial I(t)}{\partial t}+\frac{1}{i}[I(t), H(t)]=0
$$

and the eigenvalue equation of the time-dependent invariant is given by

$$
I(t)\left|\lambda_{n}, t\right\rangle=\lambda_{n}\left|\lambda_{n}, t\right\rangle,
$$

where $\frac{\partial \lambda_{n}}{\partial t}=0$. It can be seen from the invariant equation (2.6) that $I(t)$ is the linear combination of $N, \sigma_{z}, Q$ and $Q^{\dagger}$. However, it should be emphasized that the generalized invariant theory [12] can only be applied to study the system with the quasialgebra defined in [18]. Unfortunately, there is no such quasialgebra for the TLTJCM in Eq. (2.4). Many problems have been solved in Quantum Mechanics by working in the sub-Hilbert-space corresponding to a particular eigenvalue of the Hamiltonian [19]. We show that in the case of the TLTJCM, a generalized quasialgebra, which enables one to obtain the complete set of the exact solutions for the TLTJCM, can also be found by working in a sub-Hilbert-space corresponding to a particular eigenvalue of the supersymmetric generator $N^{\prime}$.

Using $a^{3}\left(a^{\dagger}\right)^{3}|m\rangle=\frac{(m+3) !}{m !}|m\rangle$ and $\left(a^{\dagger}\right)^{3} a^{3}|m+3\rangle=\frac{(m+3) !}{m !}|m+3\rangle$, one can arrive at

$$
N^{\prime}\left(\begin{array}{c}
|m\rangle \\
|m+3\rangle
\end{array}\right)=\lambda_{m}\left(\begin{array}{c}
|m\rangle \\
|m+3\rangle
\end{array}\right)
$$

with $\lambda_{m}=\frac{(m+3) !}{m !}$. One thus obtains the supersymmetric quasialgebra $\left(N, Q, Q^{\dagger}, \sigma_{z}\right)$ in the sub-Hilbert-space corresponding to the particular eigenvalue $\lambda_{m}$ of $N^{\prime}$, by replacing the generator $N^{\prime}$ with $\lambda_{m}$ in the commutation relations of Eq. (2.4), namely,

$$
\left[Q^{\dagger}, Q\right]=\lambda_{m} \sigma_{z}, \quad\left\{Q^{\dagger}, Q\right\}=\lambda_{m}, \quad\left(Q^{\dagger}-Q\right)^{2}=-\lambda_{m}
$$


In accordance with the invariant theory, we chose the invariant $I(t)$ to be of the form

$$
I(t)=-\frac{\sin \theta}{\lambda_{m}^{\frac{1}{2}}}\left[\exp (-i \phi) Q+\exp (i \phi) Q^{\dagger}\right]+\cos \theta \sigma_{z},
$$

where $\theta$ and $\phi$ are time-dependent parameters. Substitution of the expressions (2.5) and (2.10) for $I(t)$ and $H(t)$ into Eq. (2.6) leads to the following set of auxiliary equations

$$
\begin{aligned}
\dot{\theta} \cos \theta \exp (-i \phi)-i \dot{\phi} \sin \theta \exp (-i \phi)+i\left[\left(3 \omega-\omega_{0}\right) \sin \theta \exp (-i \phi)-2 g \lambda_{m}^{\frac{1}{2}} \cos \theta\right] & =0, \\
\dot{\theta}-i \lambda_{m}^{\frac{1}{2}}\left[g \exp (i \phi)-g^{*} \exp (-i \phi)\right] & =0,
\end{aligned}
$$

where the dot denotes the time derivative. The two time-parameters $\theta$ and $\phi$ in $I(t)$ are determined by these two auxiliary equations.

Using the invariant-related unitary transformation method [12], we define the unitary transformation operator as follows

$$
V(t)=\exp \left[\beta(t) Q-\beta^{*}(t) Q^{\dagger}\right]
$$

with $\beta^{*}(t)$ being the complex conjugation of $\beta(t)$. With the help of the commutation relations (2.4) and by the complicated and lengthy computations, it can be found that if $\beta(t)$ and $\beta^{*}(t)$ satisfy the following equations

$$
\beta=-\frac{\frac{\theta}{2} \exp (-i \phi)}{\lambda_{m}^{\frac{1}{2}}}, \quad \beta^{*}=-\frac{\frac{\theta}{2} \exp (i \phi)}{\lambda_{m}^{\frac{1}{2}}},
$$

then the following time-independent invariant $I_{V}$ can be obtained

$$
I_{V} \equiv V^{\dagger}(t) I(t) V(t)=\sigma_{z} .
$$

Correspondingly, the Hamiltonian (2.5) can be transformed into

$$
\begin{aligned}
H_{V}(t) \equiv & V^{\dagger}(t) H(t) V(t)-V^{\dagger}(t) i \frac{\partial}{\partial t} V(t) \\
= & \omega N+\frac{\omega}{2}\left(\sigma_{z}-1\right)+\left\{-\frac{1}{2} \lambda_{m}^{\frac{1}{2}}\left[g \exp (i \phi)+g^{*} \exp (-i \phi)\right] \sin \theta+\right. \\
& \left.+\frac{\omega_{0}-3 \omega}{2} \cos \theta-\frac{\dot{\phi}}{2}(1-\cos \theta)\right\} \sigma_{z} .
\end{aligned}
$$

by using the Baker-Campbell-Hausdorff formula [20]

$$
V^{\dagger}(t) \frac{\partial}{\partial t} V(t)=\frac{\partial}{\partial t} L+\frac{1}{2 !}\left[\frac{\partial}{\partial t} L, L\right]+\frac{1}{3 !}\left[\left[\frac{\partial}{\partial t} L, L\right], L\right]+\frac{1}{4 !}\left[\left[\left[\frac{\partial}{\partial t} L, L\right], L\right], L\right]+\cdots
$$

with $V(t)=\exp [L(t)]$. Under this unitary transformation2.12 the time-dependent Schrödinger equation (2.2) is then transformed into the following form

$$
i \frac{\partial\left|\lambda_{n}, t\right\rangle_{s 0}}{\partial t}=H_{V}(t)\left|\lambda_{n}, t\right\rangle_{s 0}
$$

where

$$
|\Psi(t)\rangle_{s}=V(t)\left|\lambda_{n}, t\right\rangle_{s 0} .
$$

One may show that the particular solution $\left|\lambda_{n}, t\right\rangle_{s}$ of Eq.(2.2) differs from the eigenfunction $\left|\lambda_{n}, t\right\rangle$ of the invariant $I(t)$ only by a phase factor $\exp \left[i \phi_{n}(t)\right]$. Then the general solution of the Schrödinger equation (2.2) can be written as

$$
|\Psi(t)\rangle_{s}=\sum_{n} C_{n} \exp \left[i \phi_{n}(t)\right]\left|\lambda_{n}, t\right\rangle,
$$

where 


$$
\begin{gathered}
\phi_{n}(t)=\int_{0}^{t}\left\langle\lambda_{n}, t^{\prime}\left|i \frac{\partial}{\partial t^{\prime}}-H\left(t^{\prime}\right)\right| \lambda_{n}, t^{\prime}\right\rangle d t^{\prime}, \\
C_{n}=\left\langle\lambda_{n}, t=0 \mid \Psi(t=0)\right\rangle_{s} .
\end{gathered}
$$

It is easy to verify that the particular solution $\left|\lambda_{n}, t\right\rangle_{s 0}$ of the time-dependent Schrödinger equation (2.17) is different from the eigenfunction $\left|\lambda_{n}\right\rangle$ of $I_{V}$ only by the same phase factor $\exp \left[i \phi_{n}(t)\right]$ as that in Eq.(2.19), i.e.,

$$
\left|\lambda_{n}, t\right\rangle_{s 0}=\exp \left[i \phi_{n}(t)\right]\left|\lambda_{n}\right\rangle .
$$

Substitution of $\left|\lambda_{n}, t\right\rangle_{s 0}$ of Eq. (2.17) into Eq. (2.21) yields

$$
-\dot{\phi}(t)\left|\lambda_{n}\right\rangle=H_{V}(t)\left|\lambda_{n}\right\rangle,
$$

which means that $H_{V}(t)$ differs from $I_{V}(t)$ only by a time-dependent multiplying c-number factor. Then the particular solution of Eq. (2.17) can be easily obtained by calculating the phase from Eq. (2.22).

The eigenstates of $\sigma_{z}$ corresponding to the eigenvalue $\sigma=+1$ and $\sigma=-1$ are $\left(\begin{array}{l}1 \\ 0\end{array}\right)$ and $\left(\begin{array}{l}0 \\ 1\end{array}\right)$, and the eigenstate of $N^{\prime}$ is $\left(\begin{array}{c}|m\rangle \\ |m+3\rangle\end{array}\right)$ corresponding to Eq. (2.8). From Eq. (2.20), (2.21), (2.22), we obtain two particular solutions of the time-dependent Schrödinger equation of the TLTJCM which can be written in the following forms

$$
\left|\Psi_{m, \sigma=+1}(t)\right\rangle_{s}=\exp \left\{\frac{1}{i} \int_{0}^{t}\left[\dot{\varphi}_{d, \sigma=+1}\left(t^{\prime}\right)+\dot{\varphi}_{g, \sigma=+1}\left(t^{\prime}\right)\right] d t^{\prime}\right\} V(t)\left(\begin{array}{c}
|m\rangle \\
0
\end{array}\right)
$$

where

$$
\begin{aligned}
\dot{\varphi}_{d, \sigma=+1}\left(t^{\prime}\right)= & \left(m+\frac{3}{2}\right) \omega\left(t^{\prime}\right)-\frac{1}{2} \lambda_{m}^{\frac{1}{2}}\left\{g\left(t^{\prime}\right) \exp \left[i \phi\left(t^{\prime}\right)\right]+g^{*}\left(t^{\prime}\right) \exp \left[-i \phi\left(t^{\prime}\right)\right]\right\} \sin \theta\left(t^{\prime}\right) \\
& +\frac{\omega_{0}\left(t^{\prime}\right)-3 \omega\left(t^{\prime}\right)}{2} \cos \theta\left(t^{\prime}\right)
\end{aligned}
$$

and

$$
\dot{\varphi}_{g, \sigma=+1}\left(t^{\prime}\right)=-\frac{\dot{\phi}\left(t^{\prime}\right)}{2}\left[1-\cos \theta\left(t^{\prime}\right)\right]
$$

and

$$
\left|\Psi_{m, \sigma=-1}(t)\right\rangle_{s}=\exp \left\{\frac{1}{i} \int_{0}^{t}\left[\dot{\varphi}_{d, \sigma=-1}\left(t^{\prime}\right)+\dot{\varphi}_{g, \sigma=-1}\left(t^{\prime}\right)\right] d t^{\prime}\right\} V(t)\left(\begin{array}{c}
0 \\
|m+3\rangle
\end{array}\right)
$$

where

$$
\begin{aligned}
\dot{\varphi}_{d, \sigma=-1}\left(t^{\prime}\right)= & \left(m+\frac{3}{2}\right) \omega\left(t^{\prime}\right)+\frac{1}{2} \lambda_{m}^{\frac{1}{2}}\left\{g\left(t^{\prime}\right) \exp \left[i \phi\left(t^{\prime}\right)\right]+g^{*}\left(t^{\prime}\right) \exp \left[-i \phi\left(t^{\prime}\right)\right]\right\} \sin \theta\left(t^{\prime}\right) \\
& -\frac{\omega_{0}\left(t^{\prime}\right)-3 \omega\left(t^{\prime}\right)}{2} \cos \theta\left(t^{\prime}\right)
\end{aligned}
$$

and

$$
\dot{\varphi}_{g, \sigma=-1}\left(t^{\prime}\right)=\frac{\dot{\phi}\left(t^{\prime}\right)}{2}\left[1-\cos \theta\left(t^{\prime}\right)\right] .
$$

Generally speaking, in Quantum Mechanics, solution with chronological-product operator (time-order operator) $P$, namely, where the time-evolution operator $U(t)=P \exp \left[\frac{1}{i} \int_{0}^{t} H\left(t^{\prime}\right) d t^{\prime}\right]$, is often called the formal solution. In the present paper, however, the solution of the Schrödinger equation governing a time-dependent system is sometimes termed the explicit solution, for reasons of the fact that it does not involve time-order operator. But, on the other hand, by using Lewis-Riesenfeld invariant theory, there always exist time-dependent parameters, for instance, $\theta$ and $\phi$ in this paper which are determined by the auxiliary equations (2.11). Traditionally, when employed in experimental analysis and compared with experimental results, these nonlinear auxiliary equations should be solved often by means of numerical computation. In view of the above reasons, the concept of explicit solution is understood in a somewhat 
relative sense, namely, it can be considered explicit solution when compared with the time-evolution operator involving time-order operator; whereas, it cannot be considered completely explicit solution for it is expressed in terms of some time-dependent parameters which should be obtained via the auxiliary equations. Hence, conservatively speaking, we regard the solution of the time-dependent system presented in the paper as exact solution rather than explicit solution.

These above two particular solutions of the Schrödinger equation (2.2) contain the corresponding dynamical phase factor $\exp \left[\frac{1}{i} \int_{0}^{t} \dot{\varphi}_{d, \sigma}\left(t^{\prime}\right) d t^{\prime}\right]$ and geometric phase factor $\exp \left[\frac{1}{i} \int_{0}^{t} \dot{\varphi}_{g, \sigma}\left(t^{\prime}\right)\right] d t^{\prime}$ with $\sigma= \pm 1$. Apparently, it can be seen that the former phase factor depends on the transition frequency $\omega_{0}(t)$ and the mode frequency $\omega(t)$ as well as the coupling coefficients $g(t)$ and $g^{*}(t)$, whereas the latter is immediately independent of these frequencies and the coupling coefficients.

One of the theoretical applications of the exact solution of time-dependent Schrödinger equation is constructing the time-dependent coherent state [21]. For instance, one result in this paper may be given as follows

$$
\begin{aligned}
\left|\Phi_{\sigma=+1}(t)\right\rangle & =\exp \left(-\frac{\xi^{2}}{2}\right) \sum_{m=0}^{\infty} \frac{\xi^{m}}{\sqrt{m !}}\left|\Psi_{m, \sigma=+1}(t)\right\rangle_{s} \\
& =\exp \left(-\frac{\xi^{2}}{2}\right) \sum_{m=0}^{\infty} \frac{\xi^{m}}{\sqrt{m !}} \exp \left\{\frac{1}{i} \int_{0}^{t}\left[\dot{\varphi}_{\sigma=+1}\left(t^{\prime}\right)\right] d t^{\prime}\right\} V(t)\left(\begin{array}{c}
|m\rangle \\
0
\end{array}\right)
\end{aligned}
$$

with $\xi$ being a time-independent parameter and $\dot{\varphi}_{\sigma=+1}(t)=\dot{\varphi}_{d, \sigma=+1}(t)+\dot{\varphi}_{g, \sigma=+1}(t)$. Time-dependent coherent state is believed to be useful in investigating the classical properties of supersymmetric Jaynes-Cummings model in quantum optics.

\section{DISCUSSION UNDER THE ADIABATIC APPROXIMATION}

The geometric phase factor in the adiabatic evolution of quantum systems was first discussed by Berry [22]. Simon then showed that this phase is in connection with a holonomy of the connection in the Hermitian line bundle over the parameter space [23]. This adiabatic phase, referred to as the Berry phase, has attracted many attentions of the investigators in various branches of physics [24]. Here we investigate the cases of the adiabatic limit. Under the adiabatic limit, we assume that the time derivative of $\theta$ vanishes, namely,

$$
\dot{\theta}=0,
$$

then the following equations can be derived from the auxiliary equations (2.11)

$$
g=|g| \exp (-i \phi), \quad g^{*}=|g| \exp (i \phi), \quad\left(3 \omega-\omega_{0}-\dot{\phi}\right) \sin \theta=2|g| \lambda_{m}^{\frac{1}{2}} \cos \theta .
$$

Inserting Eqs. (3.2) into Eq. (2.10), we obtain

$$
I(t)=\frac{-2 \cos \theta}{3 \omega-\omega_{0}-\dot{\phi}}\left[g(t) Q+g^{*}(t) Q^{\dagger}+\frac{-1}{2}\left(3 \omega-\omega_{0}-\dot{\phi}\right) \sigma_{z}\right] .
$$

In accordance with the definition of an invariant, i.e., Eq. (2.6), one can draw a conclusion that an invariant can be regarded as the Hamiltonian of the adiabatic-evolution system. Making a comparison between Eq. (2.5) and Eq. (3.3), one can see that if

$$
\dot{\phi}=\omega
$$

the following relation between $H(t)$ and $I(t)$ can be obtained

$$
H(t)=\omega(t) N-\frac{\omega(t)}{2}-\frac{2 \omega-\omega_{0}}{2 \cos \theta} I(t) .
$$

Further analysis shows that for the general three-generator Lie-algebraic systems, the invariant $I(t)$ is just considered the Hamiltonian $H(t)$ which can be expressed as

$$
H(t) \sim I(t)
$$


in the adiabatic-evolution process. According to Eq. (2.6) and Eq. (2.7), the generator $N^{\prime}$ is a time-independent invariant, while $I(t)$ is a time-dependent invariant. Since the eigenstate of $N^{\prime}$ can be rewritten as

$$
\left(\begin{array}{c}
|m\rangle \\
|m+3\rangle
\end{array}\right)=|m\rangle\left(\begin{array}{l}
1 \\
0
\end{array}\right)+|m+3\rangle\left(\begin{array}{l}
0 \\
1
\end{array}\right)
$$

which is apparently not the eigenstate of $I(t)$, even in the adiabatic evolution, the problem of eigenvalue is not very necessary as in the stationary Schrödinger equation. An invariant which satisfies Eq. (2.6) is just a conserved operator. The product of two invariants, e.g., $I(t) N^{\prime}$ is also an invariant [12]. More invariants can be constructed in terms of

$$
O(t)=U^{\dagger}(t) O U(t)
$$

where $O$ is an ordinary operator and $U(t)$ is the time-evolution operator which is given

$$
U(t)=V(t) \exp \left\{\frac{1}{i} \int_{0}^{t}\left[\dot{\varphi}_{d, \sigma}\left(t^{\prime}\right)+\dot{\varphi}_{g, \sigma}\left(t^{\prime}\right)\right] d t^{\prime}\right\} .
$$

Here the invariant-related unitary transformation formulation provides the evolution operator with an explicit expression rather than a formal solution of equation

$$
i \frac{\partial U}{\partial t}=H(t) U
$$

It should be noted that, using Eq. (3.4) and (3.1), the geometric phases $\left.\int_{0}^{t} \dot{\varphi}_{g, \sigma}\left(t^{\prime}\right)\right] d t^{\prime}$ can be rewritten as

$$
\varphi_{g, \sigma}(t)=-\frac{\sigma}{2}(1-\cos \theta) \int_{0}^{t} \omega d t
$$

with $\sigma= \pm 1$. The geometric phases (or Berry phase) in a cycle (i.e., one round trip) is

$$
\varphi_{g, \sigma}(T)=-\frac{\sigma}{2} 2 \pi(1-\cos \theta)
$$

where $2 \pi(1-\cos \theta)$ is the solid angle over the parameter space of the invariant $I(t)$, which unfolds the geometric meanings of the phase factor. The right-hand side of Eq. (3.12) is analogous to the magnetic flux produced by a monopole of strength $-\frac{\sigma}{2}$ existing at the origin of the parameter space. This, therefore, implies that geometric phase differs from dynamical phase and it involves the global and topological properties of the time evolution of a quantum system.

\section{CONCLUDING REMARKS}

In the present paper we have constructed an invariant in the sub-Hilbert-space corresponding to a particular eigenvalue of the conserved operator (time-independent invariant) $N^{\prime}$ and obtained the exact solutions of the timedependent supersymmetric TLTJCM by making use of the invariant-related unitary transformation formulation. This formulation replaces eigenstates of the time-dependent invariants by those of the time-independent invariants through the unitary transformation. In view of the above calculation, we can see that this unitary transformation formulation has some useful applications.

The exact solutions as well as their geometric phase factors of the time-dependent single- and two-photon cases can be obtained by using the present method. Since the three-level two-mode Jaynes-Cummings model plays an important role in Quantum Optics [25], the supersymmetric structure and the exact solutions of the time-dependent three-level two-mode multiphoton JCM deserves further investigations by the formalism suggested in the present paper. It is also interesting and necessary to obtain the exact solutions of the supersymmetric TLTJCM without the rotating wave approximation, by using this invariant-related unitary transformation formulation.

Acknowledgments This project was supported by the National Natural Science Foundation of China under the project No.19775040. The authors thank S.L He for useful discussions and X.C.Gao for helpful suggestions. 
[1] E.T.Jaynes and F.W.Cummings, Proc.IEEE. 51 (1963) 89.

[2] J.H.Eberly, N.B.Narozhny and J.J.Sanchez-Mondrafon, Phys.Rev.Lett. 44 (1980) 1323; Phys.Rev. A 23 (1981) 236.

[3] M.Alexanian and S.K.Bose, Phys.Rev. A 52 (1995) 221.

[4] K.Wodkiewicz, P.L.Knight, S.J.Buckle and S.M.Barnett, Phys.Rev.A 35 (1987) 2567.

[5] A.Imamolglu and S.E.Harris, Opt. Lett. 63 (1989) 1344.

[6] C.V.Sukumar and B.Buck, Phys.Lett. A 83 (1981) 211.

[7] F.L.Kien, M.Kozierowki and T.Quany, Phys.Rev. A 38 (1988) 263.

[8] S.Singh, Phys.Rev. A 25 (1982) 3206.

[9] H.X.Lu, X.Q.Wang and Y.D.Zhang, Chin.Phys. 9 (2000) 325.

[10] H.X.Lu and X.Q.Wang, Chin.Phys. 9 (2000) 568.

[11] M.V.Berry, Proc.R.Soc.London, Ser A 392 (1982) 252.

[12] X.C.Gao, J.B.Xu and T.Z.Qian, Phys.Rev. A 44 (1991) 7016.

[13] H.Lewis and W.B.Riesenfeld, J.Math.Phys. 10 (1969) 1458.

[14] X.C.Gao, J.B.Xu and T.Z.Qian, Phys.Lett. A 152 (1991) 449.

[15] J.Fu, X.C.Gao, J.B.Xu and X.B.Zou, Acta Physica Sinica(in Chinese) 48 (1999) 1011.

[16] J.Q.Shen, H.Y.Zhu and J.Li, Acta Physica Sinica(in Chinese) 50 (2001) 1884.

[17] X.C.Gao, J.Fu and J.Q.Shen, Eur.Phys.J. C 13 (2000) 527.

[18] S.S.Mizrahi, Phys.Lett. A 138 (1989) 465.

[19] L.I.Schiff, Quantum Mechanics, 3rd ed. (McGraw-Hill Book Company, New York,1968) 234.

[20] J.Wei and E.Norman, J.Math.Phys.(N.Y) 4 (1963) 575.

[21] X.C.Gao, J.B.Xu and T.Z.Qian, Annals of Phys. 204 (1990) 235.

[22] M.V.Berry, Proc.Roy.Soc.London Ser. A 392 (1984) 45.

[23] B.Simon, Phys.Rev.Lett. 51 (1983) 2167.

[24] A.Shapere and F.Wliczek, Geometric Phase in Physics (World Scientific, Singapore, 1986).

[25] Y.Wu, Phys.Rev.A 54 (1996) 1586. 